\title{
Analysis of Ten Microsecond simulation data of SARS-CoV-2 dimeric main protease
}

\author{
Md. Rimon Parves, Yasir Mohamed Riza, Shafi Mahmud' Rajib Islam' Sinthyia Ahmed, Bibi \\ Ashiana Evy, Md. Hasanuzzaman, Mohammad A Halim
}

The authors have withdrawn this manuscript because of a violation of research ethics. Unknown to the first author, the corresponding author - the main designer of the project - did not obtain consent for the use of a data-set. Therefore, the authors do not wish this work to be cited as reference for the project. If you have any questions, please contact the corresponding author" 